\title{
Aktuelles aus dem Ressort Tarife und Verträge
}

\section{Ernst Gähler ${ }^{a}$, Irène Marty ${ }^{b}$}

a Dr. med., Vizepräsident FMH, Verantwortlicher Ressort Tarife und Verträge

b Bereichsleiterin Tarife und Verträge

\section{TARMED Suisse}

Das Leitungsgremium von TARMED Suisse hat am 15. September 2010 entschieden, die neue TARMEDVersion 1.08 vorerst nicht dem Bundesrat zur Genehmigung einzureichen, da die Kosten-Nutzen-Überlegungen gegen eine Einführung sprechen. Die Einführung einer neuen Version wird deshalb um ein Jahr verschoben und voraussichtlich am 1. März 2012 in Kraft treten.

\section{Rechnungsprüfungen/Rückweisungen von Kassen}

Im Ressort Tarife und Verträge beobachten wir in den letzten Wochen wieder verstärkt, dass viele Ärzte Probleme haben mit Rechnungsprüfungen und Rückweisungen von Rechnungen durch einige Krankenkassen. Diese informieren oft gleichzeitig auch noch die Patienten, was zu Verunsicherungen führt.

Häufig geht es dabei um Probleme rund um die Dignität (Leistungen im Besitzstand) oder die Spartenanerkennung (wie Praxis-OP oder delegierte Psychotherapie). Informationen, was genau in einem solchen Fall zu tun ist und wie das Prozedere ablaufen soll, finden Sie in Heft 15/2010 der Schweizerischen Ärztezeitung [1].

Es gibt aber auch «Neuerscheinungen». Die wichtigsten neuen Gründe für Rückweisungen von einzelnen Kassen werden nachfolgend kommentiert.

\section{Kumulationsverbot von Leistungen «am gleichen Tag»}

Eine Kasse veranlasst systematische, durch das Programm generierte Rückweisungen von Tarifpositionen mit Kumulationsverbot («nicht kumulierbar mit»), die am gleichen Datum, jedoch in unterschiedlichen Sitzungen abgerechnet werden. Ein konkretes Beispiel ist die Tarifposition 00.0110 «Telefonische Konsultation durch den Facharzt» und die Position 00.0060 «Besuch». Diese beiden Leistungen wurden vom Arzt am Patienten zwar am gleichen Tag, aber zu unterschiedlichen Zeitpunkten erbracht, und demzufolge auch korrekt mit zwei unterschiedlichen Sitzungen auf der Rechnung vermerkt.

Unter den «Allgemeinen Hinweisen zum Tarif» im TARMED-Browser steht eindeutig, dass sich ein solches Kumulationsverbot zwischen zwei Leistungen immer auf eine Sitzung bezieht [2]. Der Sitzungsbegriff wird in der GI-8 definiert [3]. Die Sitzung ist die zeitliche Basiseinheit, mit der die Tarifpositionen im
TARMED in den Kostenmodellen gerechnet worden sind.

Selbstverständlich ist eine solche Abrechnung der Positionen 00.0110 «Telefonische Konsultation durch den Facharzt» und der Position 00.0060 «Besuch» am gleichen Tag möglich, die telefonische Konsultation fand am Morgen statt, der Besuch beim Patienten am Nachmittag.

Limitation der Tarifposition 39.0010 auf «1× pro Sitzung» gleich «1× pro Tag»

Ein anderes Beispiel betrifft die Radiologen in freier Praxis: Mit der Aufhebung des PIK-Entscheides I-08051-B am 1. April 2010 ist die Limitation der Tarifpositionen 39.0010/11 «Grundkonsultation/Betriebsstelle Radiologie im Röntgeninstitut ausserhalb Spital» von «1× pro Sparte und $2 \times$ pro Tag» weggefallen. Damit gilt wieder die ursprüngliche, in der TARMED-Version 01.08 enthaltene Limitation bei diesen Positionen, nämlich «1× pro Sitzung».

Eine Kasse refüsiert nun Rechnungen, auf denen diese Leistung $2 \times$ pro Tag aufgeführt ist, mit der Begründung, mit der Aufhebung des PIK-Entscheides wäre die Leistung nur noch $1 \times$ pro Tag erlaubt. Das ist natürlich falsch, die Positionen 39.0010/11 beziehen sich nun wieder auf einzelne Sitzungen und nicht auf einen Tag. Sie können deshalb am Tag mehrmals abgerechnet werden, wenn die Leistungen in verschiedenen Sitzungen (verschiedenen Sparten) erbracht wurden.

\section{Ärzte mit Leistungen im Besitzstand sind «Fachärzten» gleichzusetzen}

Eine Kasse behauptete, ein Arzt mit einer Leistung im Besitzstand sei für diese Leistung einem Facharzt gleichzusetzen; somit würden für diese Tarifposition die Facharzt-Regeln gelten. Konkretes Beispiel sind Schmerztherapeuten, die, wenn sie Positionen aus Kapitel 39 «Bildgebende Leistungen» abrechnen, keine weiteren Positionen abrechnen dürften. Für die Fachärzte für Medizinische Radiologie/Radiodiagnostik gilt diese Regel (vgl. KI-39-9 Leistungsblock LB-53) [4], jedoch nicht für andere Fachärzte. Die Regel wurde auf Fachärzte für Medizinische Radiologie/ Radiodiagnostik eingeschränkt, um Nicht-Radiologen (zum Beispiel Hausärzten) zu ermöglichen, bildgebende Leistungen zusammen mit anderen Untersuchungen in der gleichen Sitzung am Patienten zu erbringen. Sollte sich die Argumentation dieser Kasse so durch- 
setzen, müssen sich die Patienten wohl bald einen eigenen Terminkalender für ihre vielen Arztbesuche zulegen.

\section{Fazit}

Die Kassen sind zur Rechnungsprüfung verpflichtet, und die Ärzteschaft begrüsst es, wenn diese Rechnungskontrollen von den Kostenträgern auch durchgeführt werden.

Was wir vom Ressort Tarife und Verträge aber aktuell sehen und hören, geht in vielen Fällen über eine korrekte Rechnungsprüfung hinaus, ist vertraglich nicht abgesichert, stützt sich auf unhaltbare und zum Teil völlig unlogische Interpretationen, führt zu viel Aufwand und Ärger bei den Ärzten und verunsichert Patienten. Ganz zu schweigen von den Kosten, die solche «Falschprüfungen» bei den Kassen selbst verursachen, die wir als Prämienzahler dann wieder berappen dürfen.

\section{Was wir aktuell sehen und hören, geht in vielen Fällen über eine korrekte Rechnungsprüfung hinaus}

Es gilt, solchem Vorgehen einzelner Kassen Einhalt zu gebieten. Über unsere Infoline «Tarife» 0900 340340 erhalten Sie jeweils montags und mittwochs zwischen 9 und 12 Uhr kompetent Auskunft. Sie erreichen uns auch per E-Mail an: tarife@fmh.ch.

\section{Ärztliche Medikamentenabgabe}

Das FMH-Modell einer margenfreien Abgeltung der ärztlichen Medikamentenabgabe wurde im April 2010 mit einer Delegation von santésuisse besprochen. santésuisse hat an ihrer Verwaltungsratssitzung vom 25. August 2010 beschlossen, auf Verhandlungen mit der Ärzteschaft einzutreten. Die FMH erwartet einen Vorschlag für einen ersten Verhandlungstermin.

Das FMH-Modell ist kein Sparmodell, sondern beabsichtigt, die Einkünfte der Ärzte mit Bewilligung zur ärztlichen Medikamentenabgabe auf TARMED-
Positionen (AL und TL) umzulagern. Neu soll im TARMED eine Sparte «Ärztliche Medikamentenabgabe» geschaffen werden, welche die Voraussetzungen für die Abgabe zum Beispiel analog der Sparte «PraxisOP» regelt. Es darf nicht schon wieder auf dem Buckel der praktizierenden Ärzte gespart werden! Aus dem neuen System wird eine Effizienzsteigerung resultieren, die wiederum auch für die Krankenversicherer interessante Perspektiven eröffnet.

\section{QUALAB: neue Toleranzgrenzwerte ab \\ 1. Januar 2011}

In der QUALAB wurden einige Toleranzgrenzenanpassungen der internen und externen Ringversuche vorgenommen. Es betrifft folgende Parameter: Na, Bilirubin gesamt, Protein gesamt, Alanin-Aminotransferase, Amylase, HbA1C, pankreasspezifische Amylase. Die neuen Toleranzgrenzwerte sind ab dem 1. Januar 2011 gültig.

\section{Literatur}

1 Gähler E, Marty I. Tarife, Verträge und ein Brief ans BAG. Schweiz Ärztezeitung. 2010;91(15):567-9.

2 TARMED: Allgemeine Hinweise zum Tarif:

Bei Verrechnung der Tarifposition dürfen die unter «nicht kumulierbar mit» aufgeführten Leistungen/ Kapitel/Leistungsgruppen in der gleichen Sitzung nicht in Rechnung gestellt werden.

3 GI-8 Sitzung:

Eine Sitzung ist ein begrenzter Zeitraum (Kontaktaufnahme bis Kontaktende im ambulanten Bereich), während dessen ein Leistungserbringer durch einen Patienten, Paare, Familien oder Gruppen in Anspruch genommen wird. Versäumte Sitzungen gehen nicht zu Lasten der Sozialversicherungen. Die Reanimation gilt als separate Sitzung, ausgenommen IPS und Anästhesie (LB-10 und LB-52).

4 KI-39-9 Leistungsblock:

Die Tarifpositionen aus Kapitel 39 sind für Fachärzte für Medizinische Radiologie/Radiodiagnostik Teile eines Leistungsblockes und deshalb in einer Sitzung durch den gleichen Facharzt nur unter sich kumulierbar, ansonsten mit keiner anderen Tarifposition. Ausgenommen sind die Tarifpositionen Dringlichkeitszuschläge und Notfallzuschläge, Kapitel 00.08 . Weitere Ausnahmen sind ausdrücklich erwähnt. 